\title{
Changing Dogma and Decreased Collateral Damage in Breast Cancer Care
}

\author{
Jane Mendez, MD ${ }^{1}$ and Judy C. Boughey, $\mathrm{MD}^{2}$ \\ ${ }^{1}$ Miami Cancer Institute, Miami; ${ }^{2}$ Mayo Clinic, Rochester
}

Since the times of the Edwin Papyrus in 1500 BC to the present, the diagnosis and treatment of breast cancer has evolved greatly. In early times, breast cancer was incurable, and the outcomes were dismal despite the therapies with hot and cold. In the more modern era, we have transitioned from the Halstedian principles of radical surgical procedures to the systemic approach to breast cancer treatment. Why not combine surgery with adjuvant therapies, such as chemotherapy and radiation, to allow use of less radical operations? Drs. Bernard Fisher and Umberto Veronesi both challenged the dogma during their time with pivotal clinical trials, such as the National Surgical Adjuvant Breast and Bowel Project studies B-04 and B-06 and the Milan Trials. ${ }^{1,2}$ With data, many more trials, and a better understanding of tumor biology, the field of breast cancer surgery keeps evolving. We continue to question the right balance between overtreatment and undertreatment and the potential morbidities associated with such therapies. Furthermore, it is important to consider how the prescribed therapy will affect the patient's disease-free survival, overall survival, and quality of life. This issue of Annals of Surgical Oncology will provide you with thought-provoking manuscripts as we continue to challenge dogma and decrease the collateral damage of breast cancer treatment. It contains some of the scientific content as well as meeting content from the annual meeting of the American Society of Breast Surgeons (ASBrS), which was held in Las Vegas in April and had a record attendance of more than 1720 registrants from across 41 countries, including

(C) Society of Surgical Oncology 2017

First Received: 22 May 2017;

Published Online: 1 August 2017

J. Mendez, MD

e-mail: jmendez88@aol.com
232 international attendees, representing a global discussion of advances in breast cancer care.

First, what about upper extremity lymphedema related to breast cancer treatments? The ASBrS convened an international multidisciplinary consensus panel to discuss this important topic, and the manuscripts describe the recommendations from this panel and sheds some light on the prevention, diagnosis, and new treatment strategies for this potentially debilitating condition. ${ }^{3,4}$ For years, we have been advising our patients after axillary lymphadenectomy to avoid phlebotomy and blood pressure measurements on the affected arm and to use an upper extremity compression garment during air flights, but what is the evidence to support these recommendations? Also, is it possible to stratify the risk of lymphedema and identify patients who might benefit from techniques, such as axillary reverse mapping (ARM) and/or lymphatic microsurgical preventative healing approach (LYMPHA)? ARM entails mapping upper extremity lymphatics with blue dye allowing for differentiation of lymphatics draining the breast (radioactive) and the upper extremity (blue). In the only prospective study, Yue et al. randomized 265 patients to undergo axillary lymph node dissection (ALND) versus ALND + ARM. ${ }^{5}$ With 20-month follow-up, lymphedema developed in $33 \%$ of patients in the ALND group and $6 \%$ of the ALND + ARM group.

LYMPHA is a surgical approach for the primary prevention of arm lymphedema following axillary nodal dissection. The idea of LYMPHA was conceived 10 years ago, and the preliminary results were published a few years after. LYMPHA couples lymphovenous bypass (LVA) with ALND performing an anastomosis dunking the transected main lymphatic trunk(s) into a lateral branch of the axillary vein distal to a competent valve. ${ }^{6}$ Furthermore, which patients with lymphedema refractory to physical therapy and compression garments would benefit from lymph node transfer surgery? With improved understanding of the 
underlying pathophysiology of lymphedema, newer strategies both in the prevention and treatment of lymphedema continue to emerge. Also, we need to acknowledge that lymphedema may be secondary to factors other than just axillary surgery, including radiation therapy and chemotherapy, especially taxane-based chemotherapy. ${ }^{7}$

Sentinel lymph node (SLN) surgery has been the standard of care for axillary staging of the clinically negative axilla since the late 1990s. It has decreased the likelihood of upper extremity lymphedema associated with axillary surgery from $20-40$ to $5-7 \%$. One could argue that for a staging procedure, albeit lower, this is still a significant risk. ${ }^{8}$ Hence, can we identify subsets of patients where axillary surgical staging can be avoided altogether? The recent Society of Surgical Oncology Choosing Wisely guidelines recommended against SLN surgery in women older than age 70 years with hormone receptor-positive breast cancer. ${ }^{9}$ As we make treatment recommendations, it is critical to consider the patient's comorbidities, performance status, and expected prognosis as well as likelihood of nodal involvement. If the pathological findings from the SLN surgery are not going to alter the elderly patient's management, why perform the procedure? Alternatively in women with larger tumors or high-grade disease, the identification of node-positive disease is important to guide radiation decisions and fields and consideration of extended adjuvant endocrine therapy. In this issue, a model to predict likelihood of nodal positivity is presented that can be useful for patients and surgeons to identify those women age $70+$ at low risk of nodal positivity where SLN surgery may be avoided, and also to identify those women at higher risk of nodal positivity where surgical staging of the axilla may alter treatment recommendations. ${ }^{10}$

Another controversy to explore in elderly patients with favorable tumor biology treated with lumpectomy is when is it safe to omit the use of adjuvant radiation therapy. Hughes et al. addressed this question in the CALGB 9343: Lumpectomy plus tamoxifen with or without irradiation in women age 70 years or older with early breast cancer. After median follow-up of 12.6 years, a small improvement in locoregional recurrence with the addition of radiation therapy remains. However, this does not translate into an advantage in overall survival, distant disease-free survival, or breast preservation. ${ }^{11}$ Hence, in selected patients with favorable clinicopathologic characteristics, it appears that radiation therapy may be omitted safely with no differences in overall survival.

Another ongoing, controversial debate is the appropriate treatment for ductal carcinoma in situ (DCIS). The spectrum of treatment options ranges from total mastectomy to lumpectomy with radiation and endocrine therapy to no treatment at all. With improved imaging and understanding of the biology, would it be possible to identify a group of carefully selected patients with DCIS for whom surgery could be omitted? To address these concerns, there are currently three randomized, controlled trials for low-risk DCIS underway in Europe and the US designed to test the safety, efficacy, and trade-offs of active surveillance compared with usual care: LORIS (multicenter UK study), LORD (EORTC study), and COMET (comparison of operative to monitoring and endocrine therapy trial for low risk DCIS - a cooperative group US study). ${ }^{11-14}$ All three trials seek to identify a subset of patients with DCIS with low risk of both occult invasive disease at initial presentation and subsequent progression to invasive disease. The endpoint of all studies is to evaluate invasive cancer progression and quality of life. Patient selection and data from these studies will be critical for the implementation of any DCIS active surveillance program.

Another controversy in this arena is when adjuvant radiation therapy can be omitted safely in patients with DCIS. Given the favorable prognosis associated with DCIS, it is critical that we continue to question the necessary minimal intervention for maximal benefit with the lowest risk. ${ }^{15}$

As we continue to challenge dogma that changes clinical practice, are we ready in some instances to omit surgery altogether? In a thought-provoking study, Henry Kuerer et al. present data to suggest that in certain patients treated with neoadjuvant chemotherapy who have a great clinical response and have a core biopsy that demonstrates no residual disease in the breast, surgical intervention could be omitted. ${ }^{16}$ Their pilot study presents feasibility criteria to explore this question further. This is a very intriguing concept not surprising given the nature of the multidisciplinary treatment of breast cancer and all of the technological and directed-therapy treatment advances.

Despite all the exciting advances and interesting controversies, breast cancer disparities still exist. We have a better understanding that the contributing factors are not only cultural, socioeconomic, and access to care but also biological factors compound the picture. ${ }^{17}$ In her article, Lisa Newman further explores this question and looks at the global genetic admixing and its impact on triple-negative breast cancers in African American women and Hispanics. ${ }^{18}$ Are the triple-negative breast cancers that are more prevalent in younger premenopausal women more biologically aggressive? Or is it that we do not have the optimal therapy yet? From PARP inhibitors to androgen receptor inhibitors, there is significant research to discover potential cures for the triple-negative breast cancers. As we continue to unravel the biological and genetic intricacies of breast cancer, better and more targeted therapies will be discovered and disparities will be decreased.

Finally, the field of breast cancer is extremely dynamic. Challenging dogma and decreasing collateral damage with 
data have been core elements to advance the breast cancer outcomes with improved survival and good quality of life. Research, technology, and better understanding of biology continue to drive the needle forward to cure breast cancer with the ultimate goal to one day prevent and eliminate breast cancer.

\section{REFERENCES}

1. Fisher B, Andersen S, Bryant J, et al. Twenty-year follow-up of a randomized trial comparing total mastectomy, lumpectomy, and lumpectomy plus irradiation for the treatment of invasive breast cancer. $N$ Engl J Med. 2002;347(16):1233-41.

2. Veronesi U, Cascinelli N, Mariani L, et al. Twenty-year followup of a randomized study comparing breast-conserving surgery with radical mastectomy for early breast cancer. $N$ Engl $J$ Med. 2002;347(16):1227-32.

3. McLaughlin SA, Staley AC, Vicini F, et al. Considerations for clinicians in the diagnosis, prevention, and treatment of breast cancer-related lymphedema: Recommendations from a multidisciplinary expert ASBrS panel. Part 1: Definitions, assessments, education, and future directions. Ann Surg Oncol. 2017. doi:10. 1245/s10434-017-5982-4

4. McLaughlin SA, DeSnyder SM, Klimberg S, et al. Considerations for clinicians in the diagnosis, prevention, and treatment of breast cancer-related lymphedema, recommendations from an expert panel. Part 2: preventive and therapeutic options. Ann Surg Oncol. 2017. doi:10.1245/s10434-017-5964-6.

5. Ahmed M, Rubio IT, Kovacs T, Klimberg VS, Douek M. Systematic review of axillary reverse mapping in breast cancer. $\mathrm{Br} J$ Surg. 2016;103(3):170-8.

6. Yue T, Zhuang D, Zhou P, et al. A prospective study to assess the feasibility of axillary reverse mapping and evaluate its effect on preventing lymphedema in breast cancer patients. Clin Breast Cancer. 2015;15(4):301-6.

7. Nguyen TT, Hoskin TL, Habermann EB, Cheville AL, Boughey JC. Breast cancer-related lymphedema risk is related to multidisciplinary treatment and not surgery alone: results from a large cohort study. Ann Surg Oncol. 2017. doi:10.1245/s10434-0175960-x.
8. Shaitelman SF, Cromwell KD, Rasmussen JC, et al. Recent progress in the treatment and prevention of cancer-related lymphedema. CA Cancer J Clin. 2015;65(1):55-81.

9. Society of Surgical Oncology choosing wisely guidelines. http:// www.surgonc.org/docs/default-source/default-document-library/ sso-five-things-physicians-and-patients-should-question-7-112016.pdf?sfvrsn=2. Accessed 22 May 2017.

10. Welsh JL, Hoskin TL, Day CN, Habermann EB, Goetz MP, Boughey JC. Predicting nodal positivity in women 70 Years of age and older with hormone receptor-positive breast cancer to aid incorporation of a Society of Surgical Oncology choosing wisely guideline into clinical practice. Ann Surg Oncol. 2017. doi:10. 1245/s10434-017-5932-1.

11. Hughes KS, Schnaper LA, Bellon JR, et al. Lumpectomy plus tamoxifen with or without irradiation in women age 70 years or older with early breast cancer: long-term follow-up of CALGB 9343. J Clin Oncol. 2013;31(19):2382-7.

12. Francis A, Thomas J, Fallowfield L, Wallis M, et al. Addressing overtreatment of screen detected DCIS; the LORIS trial. Eur J Cancer 2015;51(16):2296-303.

13. Elshof LE, Tryfonidis K, van Leeuwen-Stok AE, et al. Feasibility of a prospective, randomised, open-label, international multicentre, phase III, non-inferiority trial to assess the safety of active surveillance for low risk ductal carcinoma in situ-the LORD study. Eur J Cancer 2015;51(12):1497-510.

14. Comparison of Operative Monitoring and Endocrine Therapy (COMET) Trial for low risk DCIS. https://clinicaltrials.gov/ct2/ show/NCT02926911. Accessed 22 May 2017.

15. Grimm LJ, Shelley Hwang E. Active surveillance for DCIS: the importance of selection criteria and monitoring. Ann Surg Oncol. 2016;23(13):4134-6. doi:10.1245/s10434-016-5596-2

16. Kuerer HM, Vrancken Peeters M-J TFD, Rea DW, Basik M, De Los Santos J, Heil J. Nonoperative management for invasive breast cancer after neoadjuvant systemic therapy: conceptual basis and fundamental international feasibility clinical trials. Ann Surg Oncol. 2017. doi:10.1245/s10434-017-5926-z.

17. Chollet-Hinton L, Anders CK, Tse CK, et al. Breast cancer biologic and etiologic heterogeneity by young age and menopausal status in the Carolina Breast Cancer Study: a case-control study. Breast Cancer Res. 2016;18(1):79.

18. Newman LA. Breast cancer disparities: socioeconomic factors versus biology. Ann Surg Oncol. 2017. doi:10.1245/s10434-0175977-1. 